\title{
Percutaneous Transvenous Embolization of Intracranial Dural Arteriovenous Fistulas with Detachable Coils and/or in Combination with Onyx
}

\author{
XIANLI LV, CHUHAN JIANG, YOUXIANG LI, XINJIAN YANG, ZHONGXUE WU \\ Beijing Neurosurgical Institute, Beijing Tiantan Hospital, Capital Medical University; Beijing, China
}

Key words: arteriovenous fistula, cavernous sinus, dural arteriovenous malformation, transvenous embolization

\section{Summary}

This study evaluated angiographic and clinical results in patients with a dural arteriovenous fistula (DAVF) who underwent percutaneous transvenous embolization.

Retrospective chart analysis and radiographic studies were performed in 23 patients (aged 1170 yrs) with a DAVF treated with percutaneous transvenous embolization in the past five years. Lesions were located in the anterior cranial fossa, cerebellar tentorium, transverse-sigmoid sinus and cavernous sinus. All procedures were analyzed with regard to presentation, delivery, angiographical and clinical outcome.

Data for 23 patients (age range, 11-70 yrs, mean age 49.5yrs) with DAVFs (cavernous sinus [CS], $n=17$; transverse-sigmoid sinus, $n=3$; anterior cranial fossa, $n=2$; cerebellar tentorium, $n=1)$ were retrospectively reviewed. The DAVFs were treated with coils or a combination with Onyx via different transvenous approaches, in 28 procedures. Cerebral angiography was performed to confirm the treatment.

The mean clinical follow-up period was 22.1 months.

Transvenous treatment of intracranial DAVFs can be safe and effective if various transvenous approaches are attempted. Percutaneous transvenous embolization with detachable platinum coils or a combination with Onyx is effective in the treatment of DAVFs.

\section{Introduction}

DAVFs represent 10 to $15 \%$ of intracranial arteriovenous malformations ${ }^{46,48,56}$. Their classification is determined on the basis of the arterial supply or the venous drainage pattern ${ }^{46,48,53,56}$. Carotid cavernous fistulae (CCFs) are classified as direct (Barrow Type A) fistulae, which are often posttraumatic high-flow shunts between the cavernous portion of the internal carotid artery (ICA) and the cavernous sinus (CS), or indirect dural fistulae (Barrow Type BD). Multiple arterial feeders arise from the ICA (Barrow Type B), the external carotid artery (ECA) (Barrow Type C), or both (Barrow Type D), with numerous microfistulae within the sinus wall ${ }^{4}$. Recently published classifications of DAVFs (e.g., Cognard's classification) are based on the pattern of drainage for estimation of bleeding risks ${ }^{8}$. Depending on the DAVFs location and the venous drainage, clinical presentations range from asymptomatic to symptomatic $^{1,7,15,16,26,36,38}$, with pulse-synchronous bruit, headaches, neurological deficits, venous hypertensive encephalopathy with dementia, or intracranial hemorrhage resulting from venous hypertension.

The decision to treat is based on the venous drainage pattern, the natural history of the lesion, the severity of presenting symptoms, the patient's general condition, angiographic features, the location of the DAVF, and the mor- 

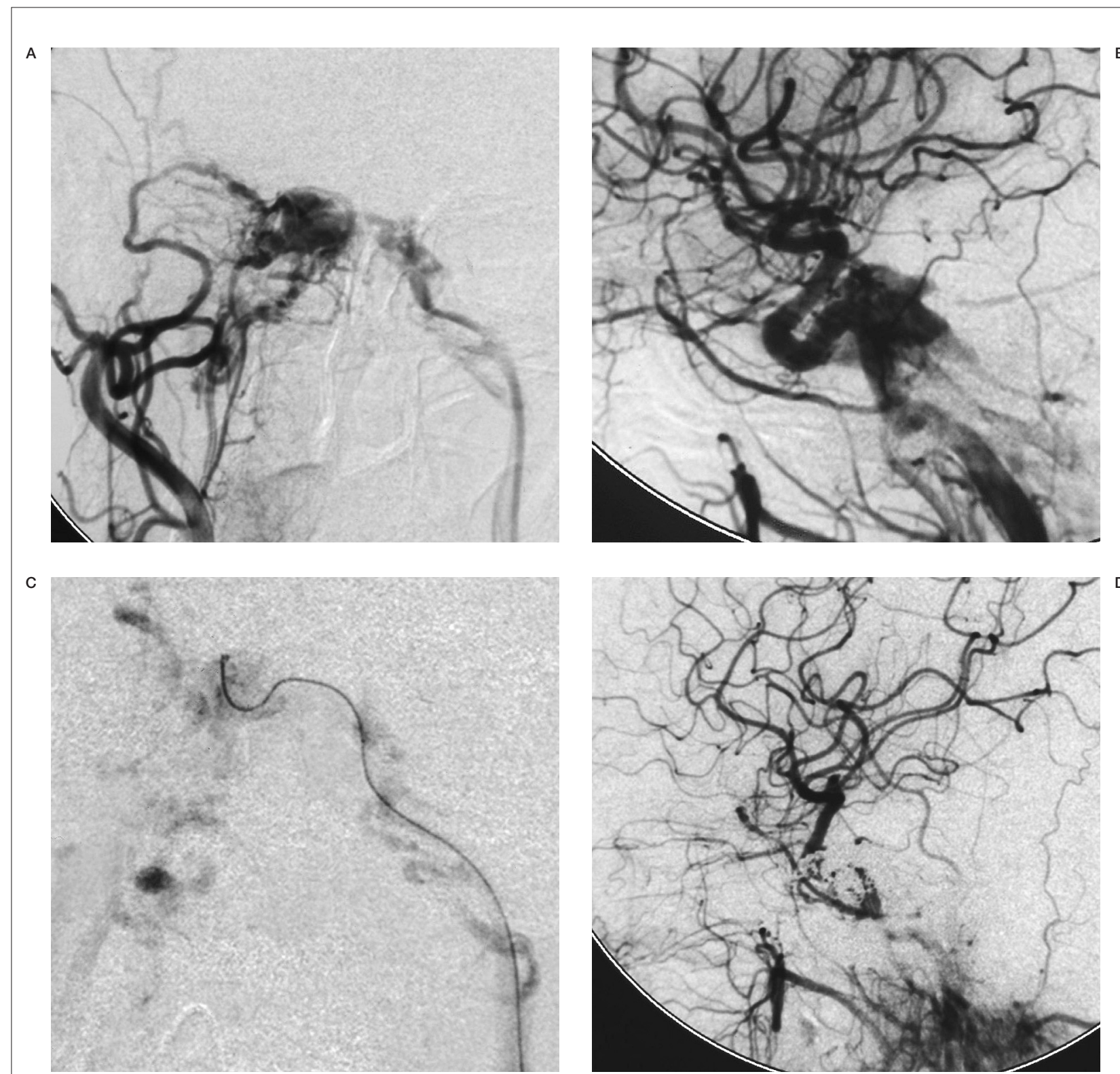

bidity and mortality rates of the procedure being considered. The goals of treatment are the prevention of risks and the elimination of symptoms caused by the arteriovenous shunt. Because of the bleeding risk, intracranial DAVFs with retrograde cortical drainage often require an anatomic cure. They can be treated with different modalities, such as endovascular procedures with transvenous and transarterial ${ }^{1,11,17,19-25,33,35,37,42,43,49-57}$, surgery $7,10,12,13,16,18,29,41,55,58$, gamma knife surgery $39,40,4,4,45,51$, or combinations of the three ${ }^{15,30,31,33}$; in selected cases, the lesions can be treated conservatively ${ }^{22}$.

Transvenous embolization with coils has be-

come the standard treatment for DAVFs ${ }^{27,47,48}$. Today, transarterial Onyx embolization has been used preoperatively or as additional therapy to prompt the cure rate. This study presents our experience of different transvenous embolization techniques for the treatment of intracranial DAVFs and transvenous administration of Onyx.

\section{Patient and Methods}

Between February 2003 and February 2008, 23 patients (nine females, 14 males) with DAVFs involving the anterior cranial fossa 

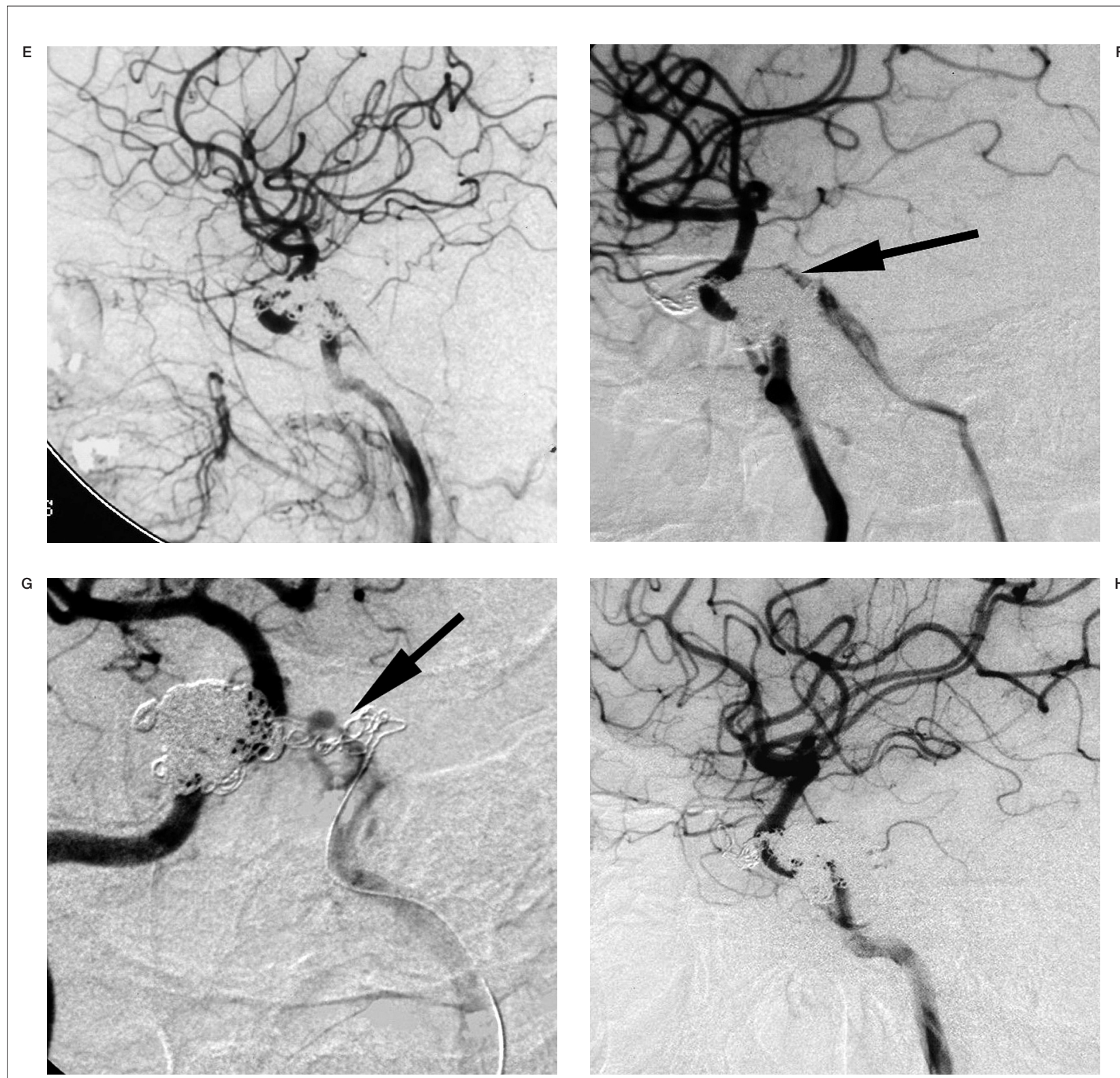

$(\mathrm{ACF})$, cerebellar tentorium, transverse-sigmoid sinus (TSS) and cavernous sinus (CS) were treated by percutaneous transvenous embolization at Beijing Tiantan Hosptial. Symptoms, locations of DAVFs, and access routes of venous embolization in each case are presented in Tables 1 and 2.

Bilateral selective ICA and ECA angiography and vertebral artery angiography were performed for all patients, for assessment of the feeding arteries, fistula sites and venous drainage. The arteriovenous shunts were approached via the venous route. We first placed 6-French sheaths in the femoral artery and

Figure 1 Patient 9, a 44-year-old woman demonstrated a cavernous DAVF of Barrow Type D/Cognard Type IIa. Right CCA angiogram, anteroposterior projection (A), left CCA angiogram, lateral projection (B) showing a CDAVF fed by both internal and external carotid arteries, mainly drained to the left InfPS and the right SOV. Frontal superselective angiogram of the right CS (C) showing the microcatheter positioned close to the fistula site. After embolization, anterograms of the right common carotid artery (lateral view, D) and left common carotid artery (lateral view, E) demonstrating complete occlusion of the fistula. F) Six months later, lateral angiogram of the right ICA showing persistent filling of the residual fistula (arrow). G) Occlusion of the intercavernous sinus (arrow) with several standard coils. H) Arteriogram of the right ICA (lateral view) after complete embolization of the right-side fistula. 


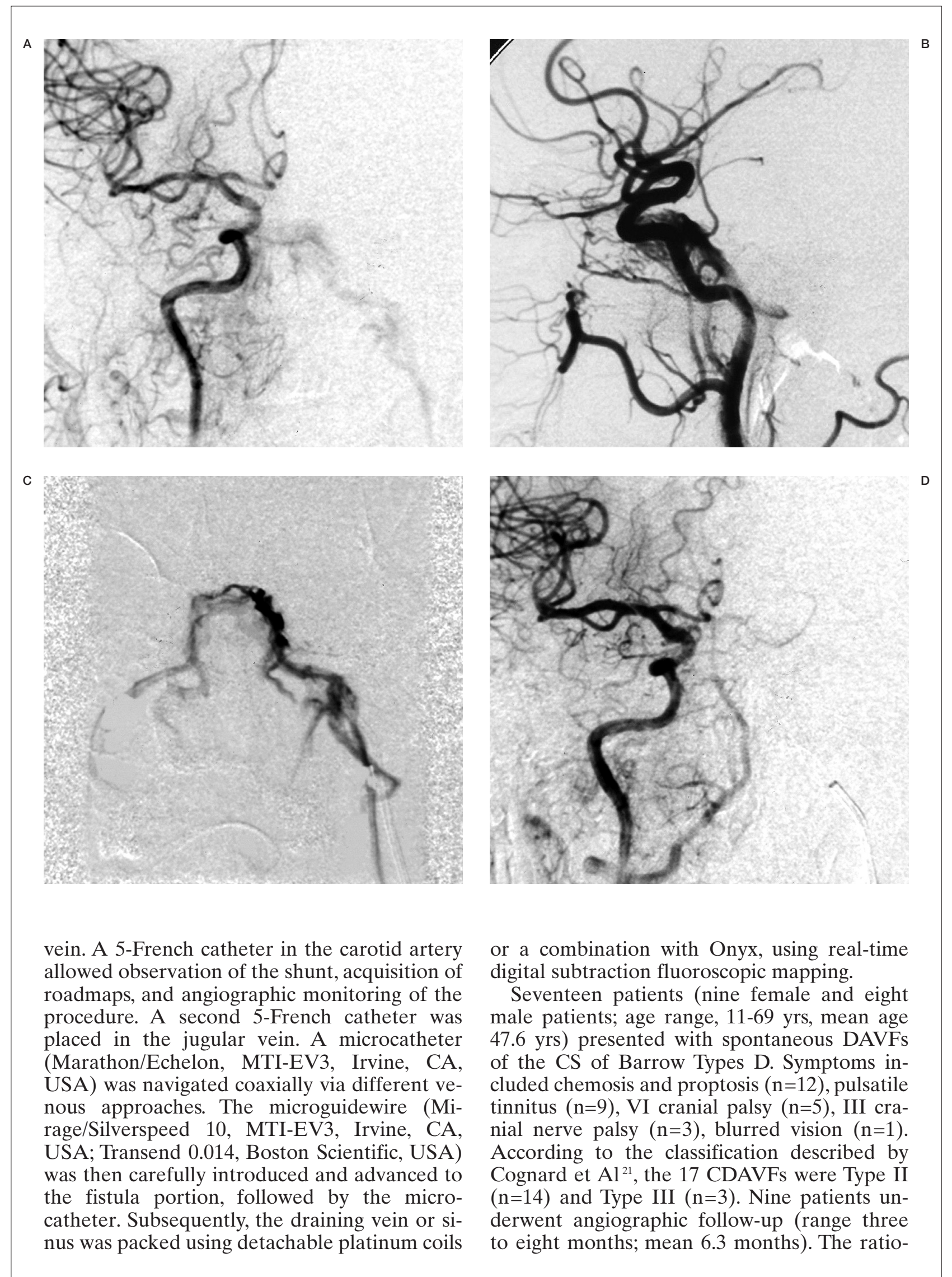




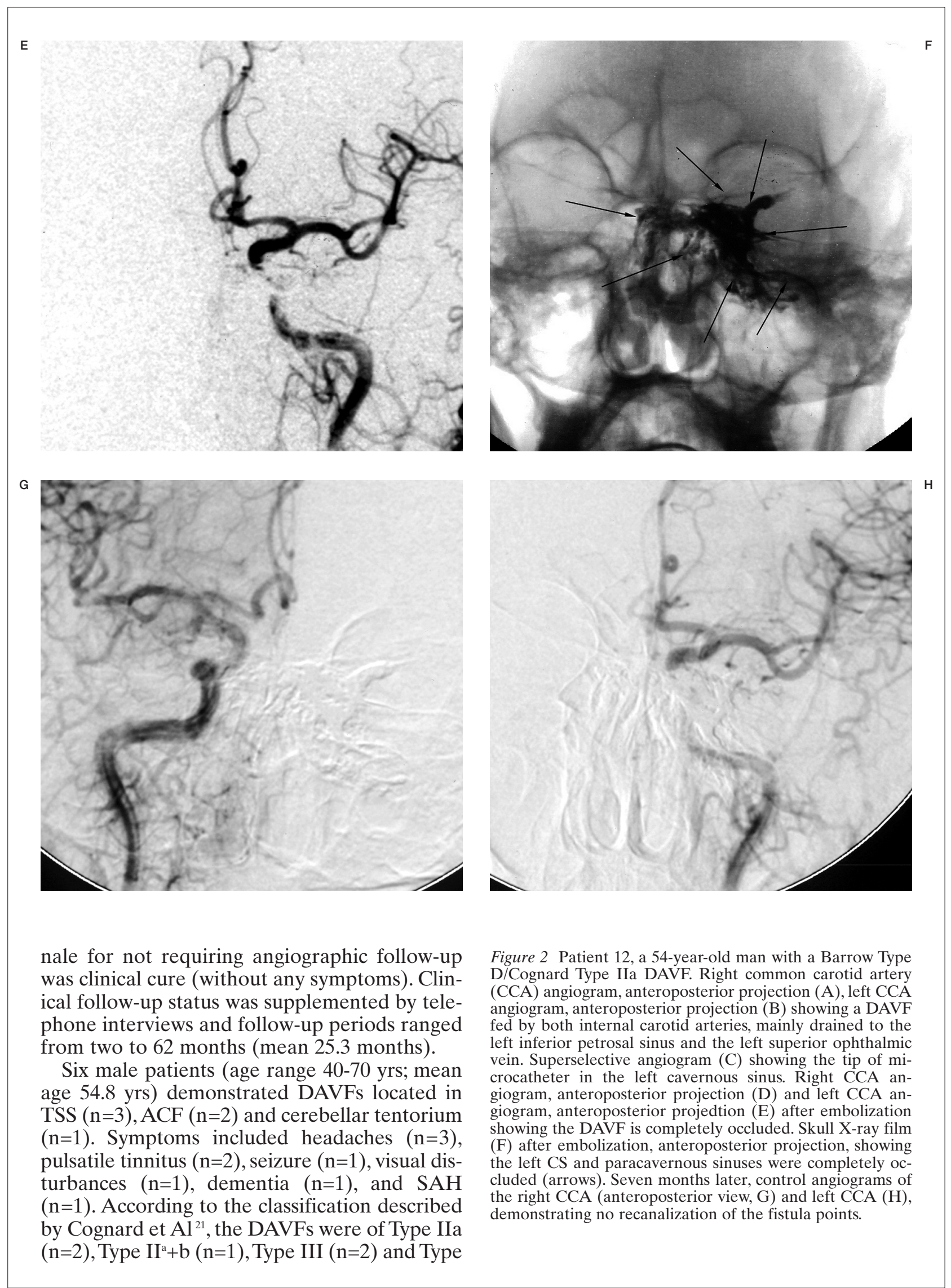




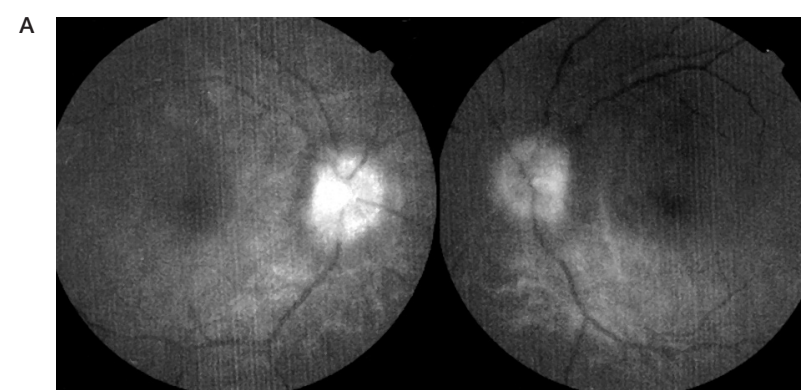

B

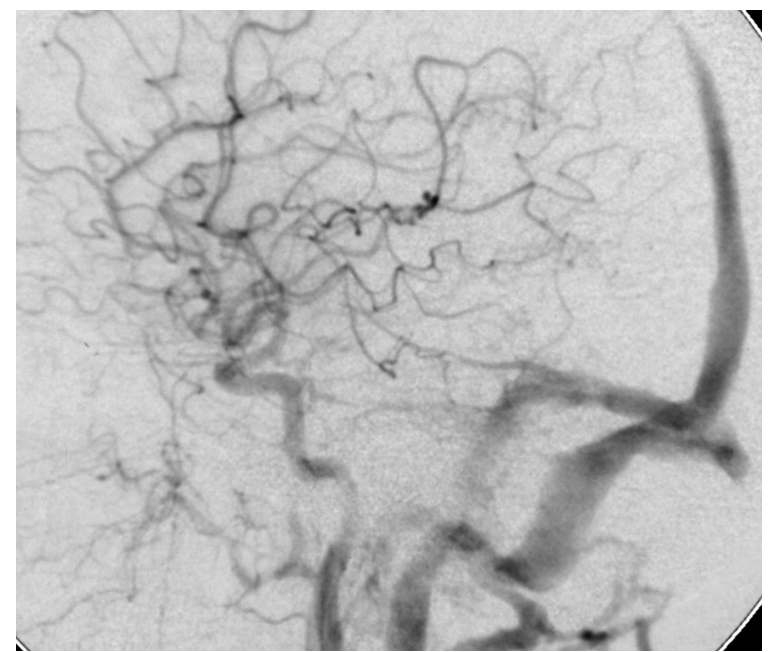

c

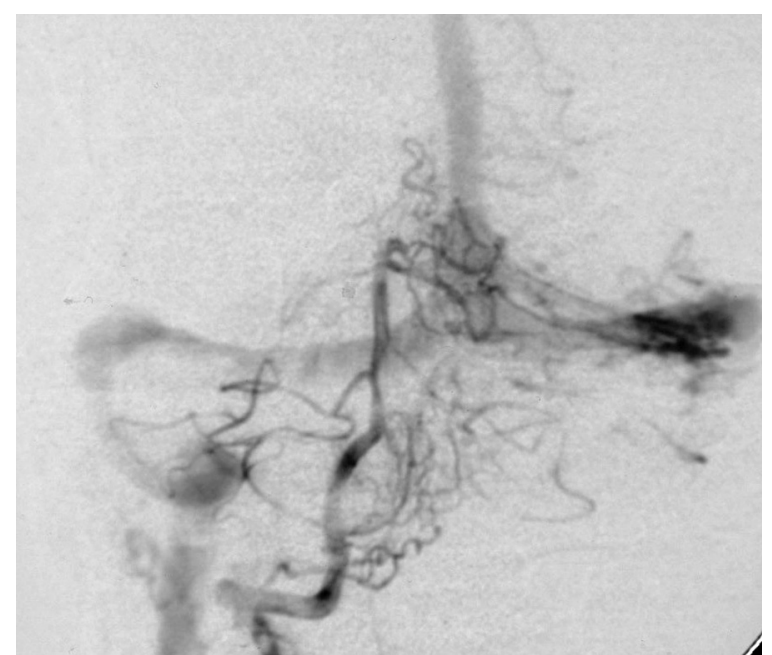

Figure 3 Patient 23, a 56-year-old man with a left Cognard Type IIa DAVF, with SSS reflux. A) An ophthalmological examination revealed bilateral papilledema. B) Arteriogram of the left CCA (arterial phase, lateral view), showing multiple feeders arising from the ICA and ECA. C) Arteriogram of the right VA (arterial phase, frontal view), showing multiple feeders arising from the VA. D) The microcatheter was navigated into the left TSS via the right IJV-TSS. E,F) Arteriograms (lateral view) of the left common carotid artery (E) and left VA. The residual fistula was Cognard Type I with parallel venous channel was coiled and the parent sinus was patent, with preservation of the venous outflow (arrows) of the temporal lobe through the vein of Labbe.
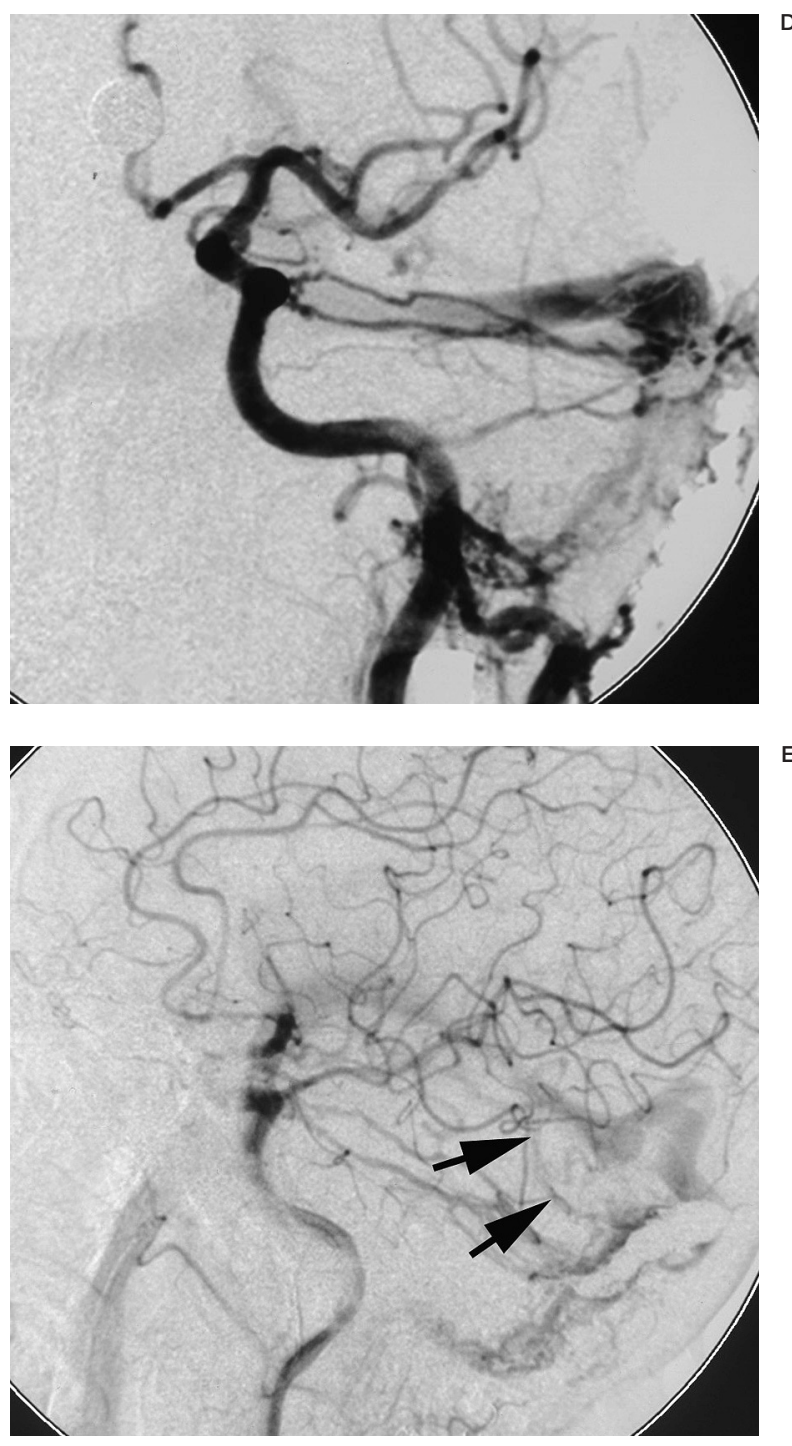

E

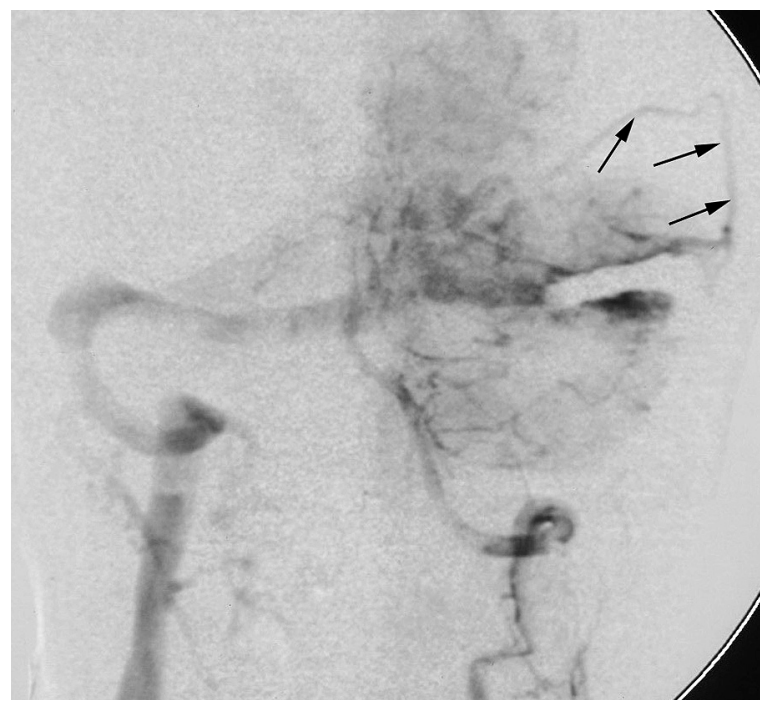


IV $(\mathrm{n}=1)$. The fistulae were spontaneous. Clinical follow-up periods for this group ranged from two to 36 months (mean, 13 months).

\section{Results}

Twenty-three patients with DAVFs were reviewed in this series. Twenty-one DAVFs were completely embolized with detachable platinum coils or a combination with Onyx. The clinical data, and the angiographic results are summarized in Tables 1 and 2 .

\section{Transvenous embolization of the CS.}

Twenty-two transvenous and two transarterial approaches were performed for the group of 17 DAVFs of the CS. Sixteen patients with DAVFs were initially treated with a transvenous route while one patient was treated with a transarterial route first. If the drainage was to the ipsilateral CS and/or predominantly posteriorly, then we first approached via the internal jugular vein (IJV)-inferior petrosal sinus (InfPS)-CS $(n=13)$. If the InfPS could be passed $(n=13)$, then the success rate was high, with complete occlusion in 16 cases. The more invasive direct SOV approach was chosen only if alternative endovascular transvenous routes had failed (one out of 17 cases) to yield complete occlusion of the fistula. The transvenous approach via the facial vein $(\mathrm{FV})$ was possible in two cases with complete occlusion of the fistula. An alternative approach via the superior petrosal sinus was performed in one case but failed.

\section{Follow-up outcome}

Sixteen of the 17 DAVFs (94.1\%) of the CS were cured anatomically and clinically. One patient $(5.9 \%)$ with initial left VIth cranial nerve palsy did not completely resolve and developed brain infarction at 30-month follow-up. We encountered complications in five cases $(29.4 \%)$ : one patient developed local alopecia on the right side due to $\mathrm{X}$-ray radiation, two patients developed reflexive bradyarrythmia and two patients demonstrated CNVI palsy after transvenous treatment. Angiographic follow-up (4-7 months; mean five months) was obtained in six patients. There was one recanalization in one patient; additional coil embolization was performed in five patients with residual fistulae. Residual aneurysm resolved in four patients with one failure.

\section{Illustrative cases}

\section{Patient 9}

A 44-year-old woman demonstrated right proptosis and IVth cranial nerve palsy and excessive pulsatile bruits. Cerebral angiography revealed a DAVF of the right CS. Angiography of the right CCA demonstrated persistent filling of the left InfPS (Figure 1A). Therefore, a transvenous approach was chosen. The microcatheter was navigated through the left InfPS and the intercavernous sinus to the right $\mathrm{CS}$ (Figure 1C). The right CS was occluded with 28 standard coils (Figure 1D,E). However, the pulsatile tinnitus was still persistent at sixmonth follow-up study. Control angiography demonstrated recurrence of the fistula (Figure $1 \mathrm{~F})$ and the same procedure was performed (Figure 1G). The residual fistula was occluded completely by another six standard coils (Figure $1 \mathrm{H})$.

\section{Patient 12}

A 54-year-old man presented with blepharotosis, diplopia and chemosis. Cerebral angiograms showed a cavernous DAVF (Figure 2A,B). Recommendation was made for transvenous embolization of this lesion. Onyx injection was performed after three standard coils (two $5 \times 12$, one $6 \times 15$ ) were introduced until control angiogram revealed complete occlusion of the DAVF. The patient's symptoms resolved within three months, there was no recanalization on seven-month follow-up angiograms (Figure 2 G,H).

\section{Transvenous embolization in other locations}

For the group of DAVFs in other locations, clinical symptoms included headaches $(n=3)$, pulsatile tinnitus $(n=2)$, seizure $(n=1)$, visual disturbances $(n=1)$, dementia $(n=1)$, and SAH $(\mathrm{n}=1)$.

Five DAVFs experienced cures, and one patient (1/6) experienced improvement. We performed six transvenous approaches usually via the IJV-saggital sinus (SS), IJV-TSS and leptomeningeal routes. The technical success rate was $100 \%$ (six out of six cases), with complete occlusion in $83.33 \%$ of cases (five out of six cases). The rate of incomplete occlusion was $16.67 \%$ (one out of six cases). For four patients, we performed the transarterial approach prior to transarterial embolization. 


\begin{tabular}{|c|c|c|c|c|c|c|c|c|c|}
\hline 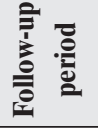 & 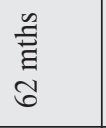 & $\begin{array}{l}\text { 竭 } \\
\text { in }\end{array}$ & $\begin{array}{l}\stackrel{n}{ \pm} \\
\stackrel{E}{E} \\
\text { in }\end{array}$ & $\begin{array}{l}\text { 氖 } \\
\text { है } \\
\text { in }\end{array}$ & 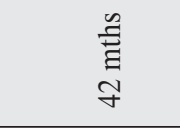 & $\begin{array}{l}\stackrel{n}{E} \\
\vec{E} \\
F\end{array}$ & 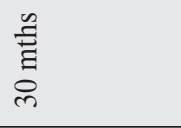 & $\begin{array}{l}\text { 气 } \\
\text { 音 } \\
\text { ¿े }\end{array}$ & $\begin{array}{l}\stackrel{n}{E} \\
\stackrel{\Xi}{\Xi} \\
\text { I }\end{array}$ \\
\hline 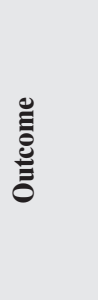 & 气 & $\stackrel{\Xi}{\Xi}$ & 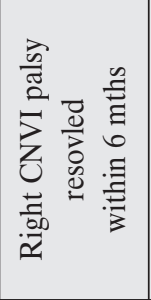 & ڤँ & 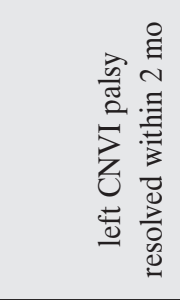 & 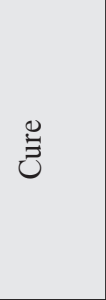 & 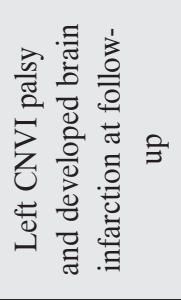 & 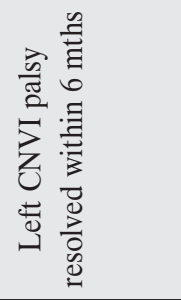 & 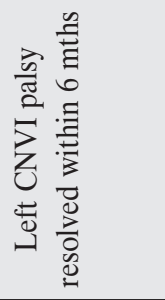 \\
\hline 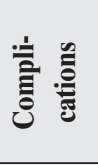 & 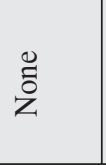 & 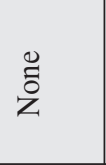 & $\begin{array}{l}\text { ̊̃̆ } \\
\text { Ż }\end{array}$ & $\begin{array}{l}0 \\
\tilde{0} \\
z\end{array}$ & $\begin{array}{ll}\stackrel{\Xi}{0} \\
\stackrel{\tilde{z}}{z}\end{array}$ & $\begin{array}{l}0 \\
\check{0}\end{array}$ & ஜ̈ & 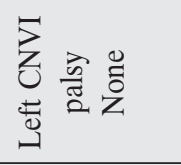 & $\begin{array}{ll}\stackrel{0}{0} \\
\stackrel{\tilde{\sigma}}{z}\end{array}$ \\
\hline 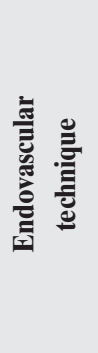 & 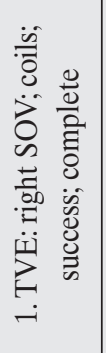 & 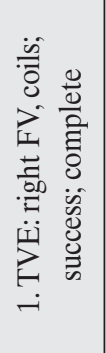 & 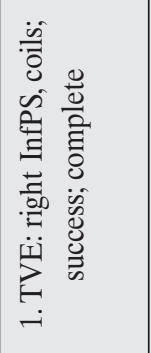 & 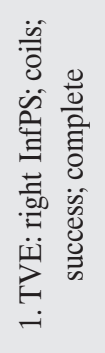 & 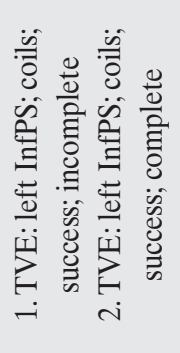 & 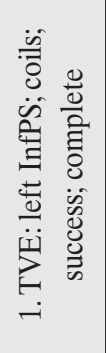 & 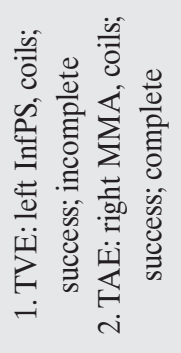 & 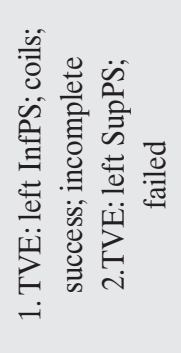 & 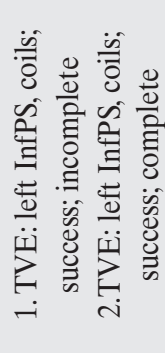 \\
\hline 旁 & 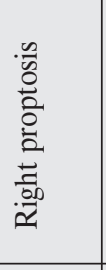 & 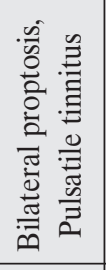 & 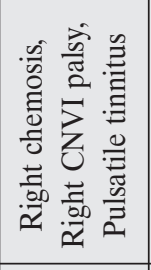 & 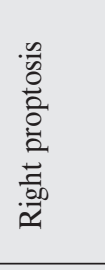 & 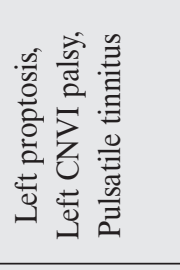 & 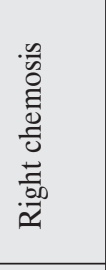 & 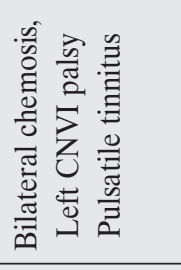 & 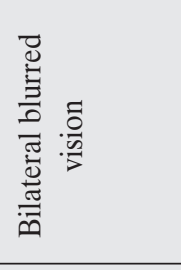 & 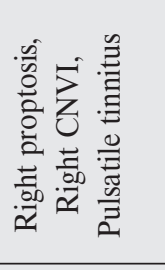 \\
\hline 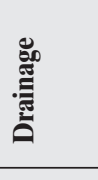 & $\begin{array}{l}\vec{z} \\
0 \\
\frac{1}{.00} \\
\vec{a} \\
\end{array}$ & $\begin{array}{l}\infty \\
0 \\
\infty \\
\tilde{0} \\
\infty \\
\end{array}$ & 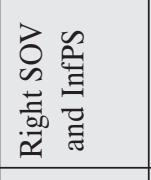 & 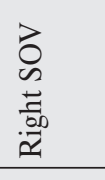 & 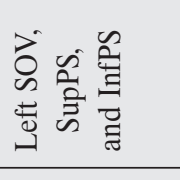 & 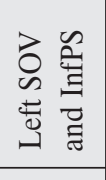 & 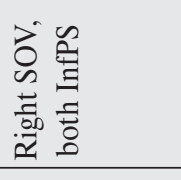 & 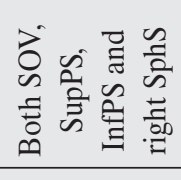 & 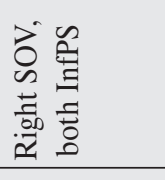 \\
\hline 遌 & 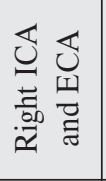 & 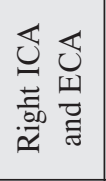 & 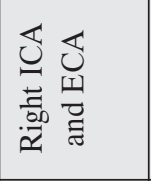 & 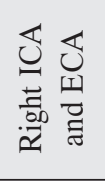 & 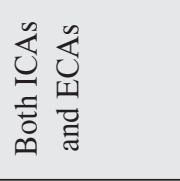 & 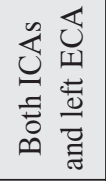 & 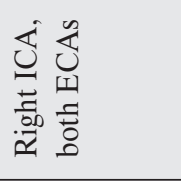 & 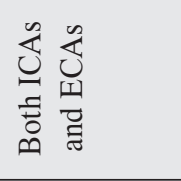 & 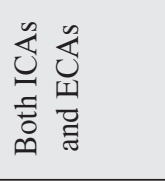 \\
\hline 浐 & 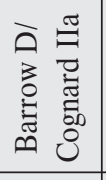 & 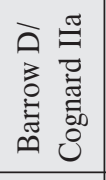 & 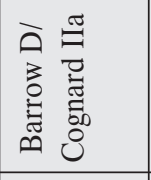 & 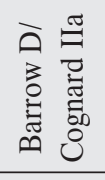 & 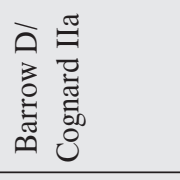 & 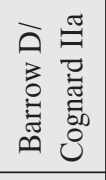 & 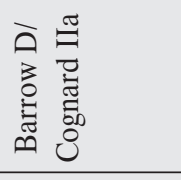 & 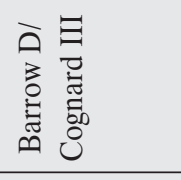 & 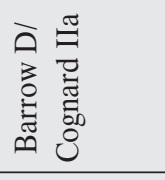 \\
\hline ฮั & $\simeq$ & $\simeq$ & $\simeq$ & \lrcorner & \lrcorner & \lrcorner & د. & $\infty$ & $\simeq$ \\
\hline 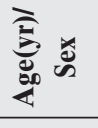 & $\underset{\Xi}{\Sigma}$ & 핑 & 孚 & $\underset{\infty}{\infty}$ & $\sum_{\exists}$ & 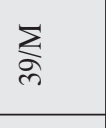 & $\frac{1}{\delta 0}$ & 秥 & 岁 \\
\hline 竞 & $-i$ & $i$ & $\dot{m}$ & $\dot{\nabla}$ & in & 0 & $\therefore$ & $\infty$ & $\sigma^{\circ}$ \\
\hline
\end{tabular}




\begin{tabular}{|c|c|c|c|c|c|c|c|c|c|}
\hline 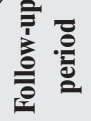 & 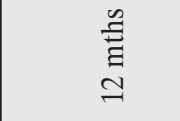 & 音 & $\begin{array}{l}\stackrel{\mathscr{E}}{\Xi} \\
\stackrel{m}{ٍ}\end{array}$ & $\begin{array}{l}\underset{\Xi}{\Xi} \\
\stackrel{\Xi}{\Xi}\end{array}$ & $\begin{array}{l}\tilde{a} \\
\vec{\Xi} \\
a\end{array}$ & 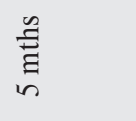 & $\begin{array}{l}\stackrel{y}{E} \\
\stackrel{\vec{g}}{\sigma}\end{array}$ & $\underset{\text { E }}{\stackrel{n}{\sim}}$ & ญै \\
\hline 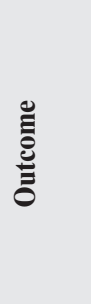 & $\stackrel{\Xi}{\Xi}$ & ڤ్ & 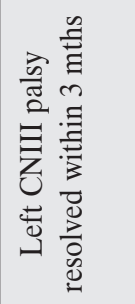 & 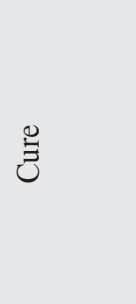 & 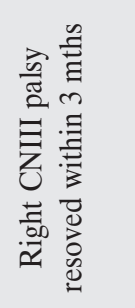 & 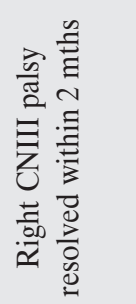 & 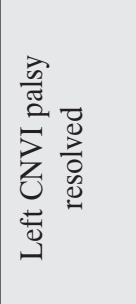 & 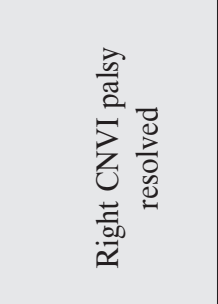 & 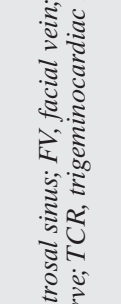 \\
\hline 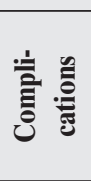 & $\begin{array}{ll}\tilde{0} & 0 \\
\check{z}\end{array}$ & 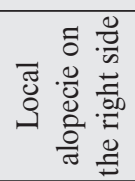 & 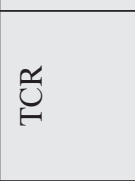 & 苂 & 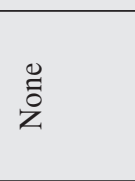 & త્రુ & 䓂 & 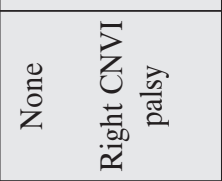 & 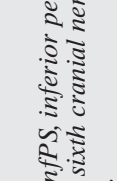 \\
\hline 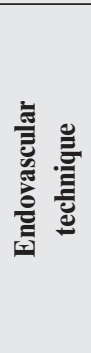 & 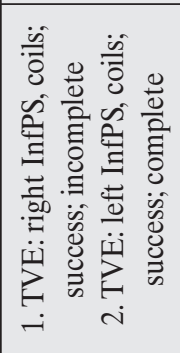 & 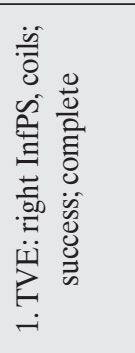 & 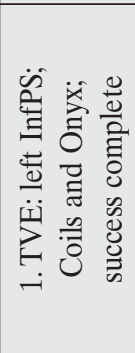 & 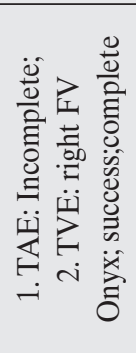 & 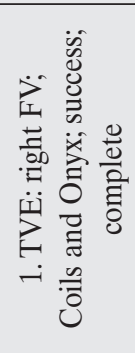 & 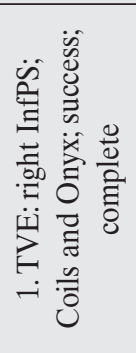 & 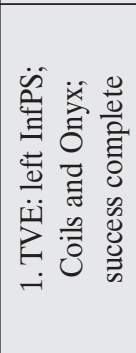 & 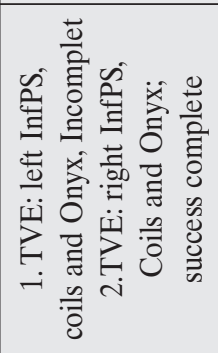 & 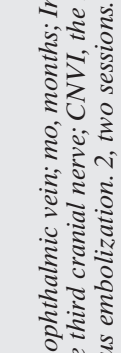 \\
\hline 言 & 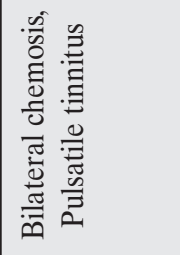 & 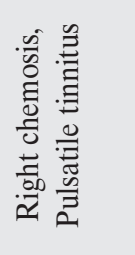 & 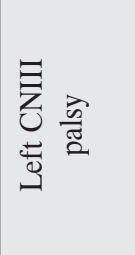 & 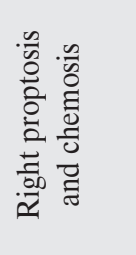 & 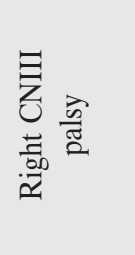 & 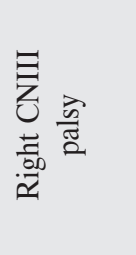 & 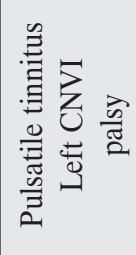 & 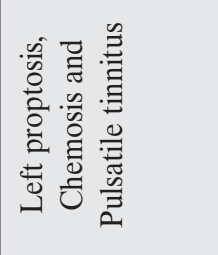 & 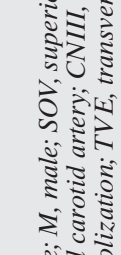 \\
\hline 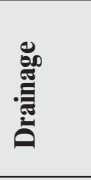 & 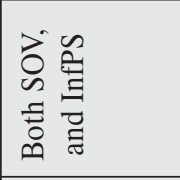 & 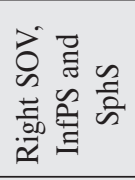 & 嵑 & $\begin{array}{l}\vec{z} \\
0 \\
\frac{0}{00} \\
\vec{a}\end{array}$ & $\begin{array}{l}\overrightarrow{0} \\
0 \\
= \\
\frac{.00}{01} \\
\alpha 1\end{array}$ & 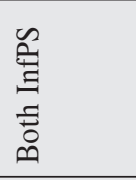 & 感 & 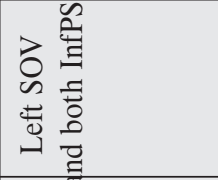 & 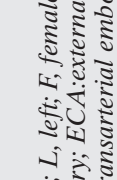 \\
\hline 离 & 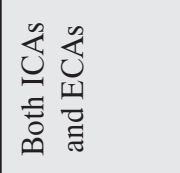 & 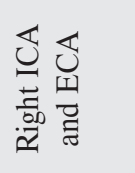 & $\begin{array}{l}\sum_{0}^{n} \\
0 \\
\overline{0} \\
0 \\
\infty\end{array}$ & 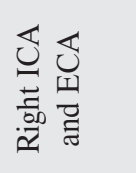 & $\begin{array}{l}\sum_{0}^{0} \\
\text { 苛 } \\
\infty\end{array}$ & 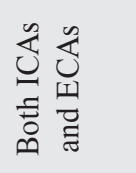 & 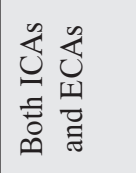 & 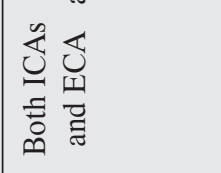 & 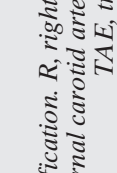 \\
\hline 农 & 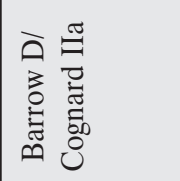 & 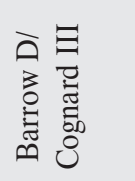 & 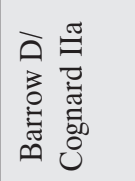 & 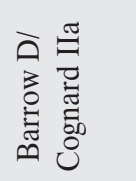 & 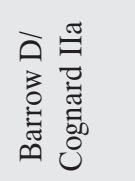 & 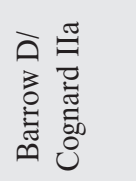 & 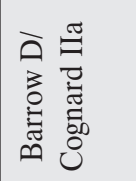 & 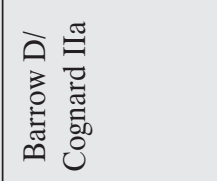 & 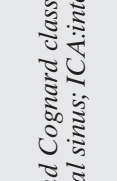 \\
\hline 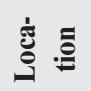 & $\infty$ & $\simeq$ & ـ & دـ & $\simeq$ & $\simeq$ & ــ & $\infty$ & 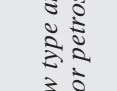 \\
\hline 愿 & 乩 & 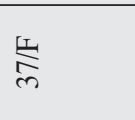 & 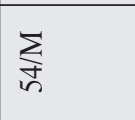 & 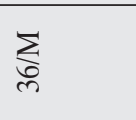 & $\frac{1}{8}$ & $\sum_{i n}$ & $\sum_{i}$ & 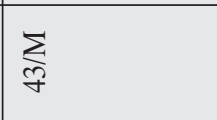 & 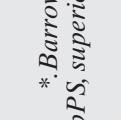 \\
\hline نे & $\stackrel{\circ}{\circ}$ & $\dot{=}$ & $\simeq$ & $\dot{\Omega}$ & $\dot{ \pm}$ & $\ddot{n}$ & $\stackrel{\leftrightarrow}{\circ}$ & $\stackrel{\check{1}}{0}$ & \\
\hline
\end{tabular}




\begin{tabular}{|c|c|c|c|c|c|c|c|}
\hline 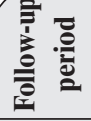 & $\begin{array}{l}\stackrel{n}{\Xi} \\
\vec{\Xi}\end{array}$ & $\underset{m}{\stackrel{n}{E}}$ & $\begin{array}{l}\stackrel{\mathscr{a}}{\Xi} \\
\stackrel{\Xi}{g}\end{array}$ & 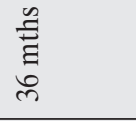 & $\begin{array}{l}\text { 号 } \\
\text { 竞 }\end{array}$ & $\underset{\tilde{E}}{\tilde{E}}$ & \multirow{11}{*}{ 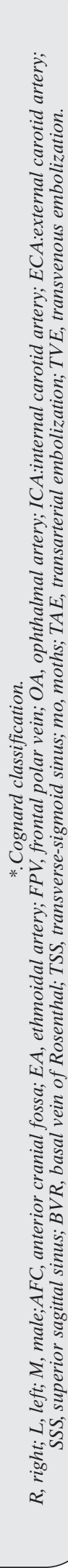 } \\
\hline $\begin{array}{l}\text { 兽 } \\
\text { 产 }\end{array}$ & 气゙ & $\stackrel{\Xi}{\Xi}$ & $\stackrel{\Xi}{\Xi}$ & $\stackrel{\Xi}{\Xi}$ & $\stackrel{\Xi}{\Xi}$ & 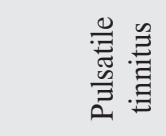 & \\
\hline 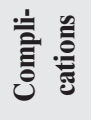 & $\begin{array}{ll}\tilde{E} & \Xi \\
z\end{array}$ & $\stackrel{\Xi}{z}$ & $\begin{array}{l}\tilde{\sigma} \\
z\end{array}$ & $\begin{array}{l}\stackrel{0}{0} \\
\check{z}\end{array}$ & $\begin{array}{ll}\ddot{0} & \ddot{0} \\
\check{z} & \stackrel{z}{z}\end{array}$ & 音 & \\
\hline 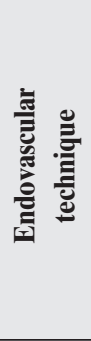 & 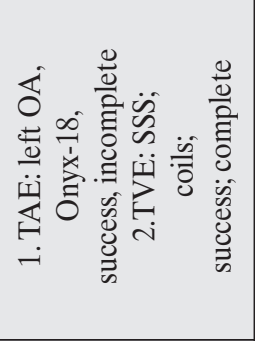 & 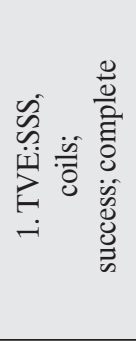 & 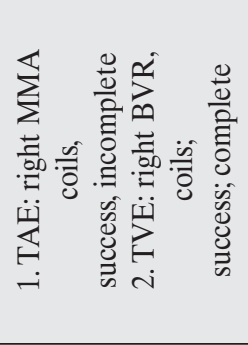 & 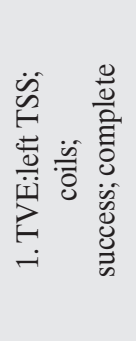 & 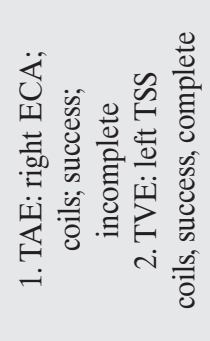 & 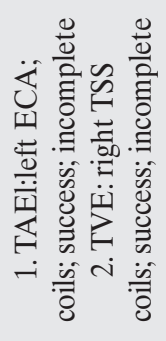 & \\
\hline 袅 & 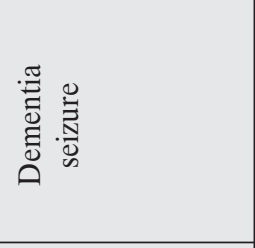 & 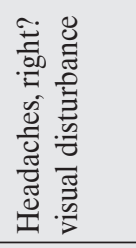 & 㞼 & 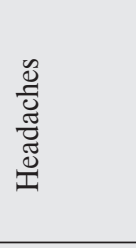 & 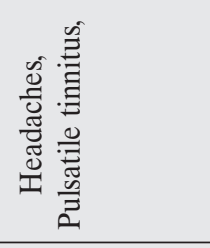 & 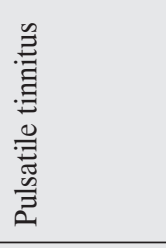 & \\
\hline 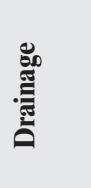 & 齐 & 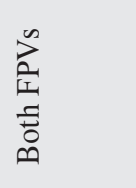 & 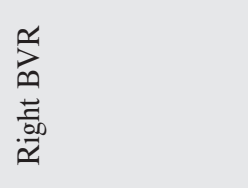 & 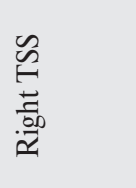 & 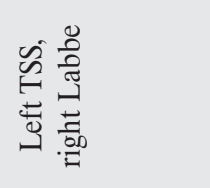 & 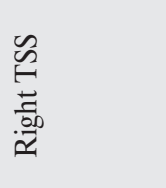 & \\
\hline 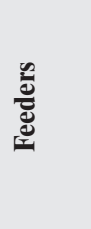 & 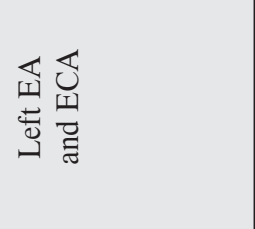 & 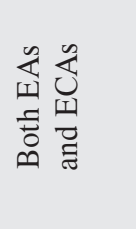 & 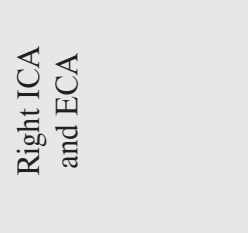 & 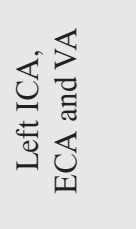 & 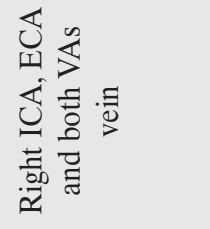 & 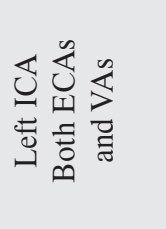 & \\
\hline 产 & $\begin{array}{l}\Xi \\
0 \\
\bar{\Xi} \\
\tilde{D}_{0} \\
8\end{array}$ & 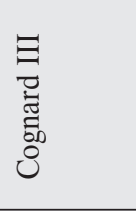 & 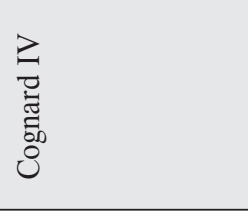 & 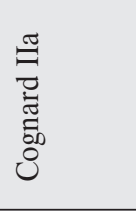 & 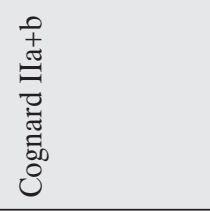 & 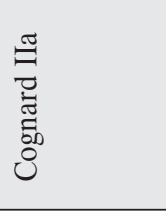 & \\
\hline פ气 & 崫 & 岂 & 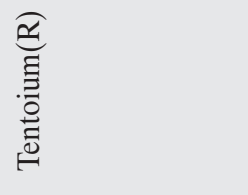 & $\begin{array}{l}\vec{E} \\
\tilde{\omega} \\
\Leftrightarrow\end{array}$ & 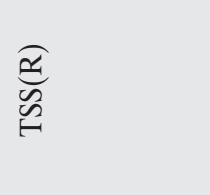 & $\begin{array}{l}\vec{\Xi} \\
w \\
\omega \\
\omega\end{array}$ & \\
\hline 產 & $\sum_{i k b}$ & 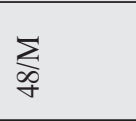 & $\sum_{\unrhd}$ & $\sum_{i=}$ & 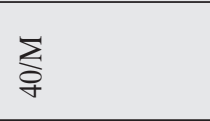 & $\sum_{i}$ & \\
\hline 产 & $\stackrel{\infty}{\rightarrow}$ & $\stackrel{2}{-}$ & $\dot{\overbrace{}}$ & $\dot{\vec{N}}$ & ત̇ & $\ddot{\lambda}$ & \\
\hline
\end{tabular}




\section{Follow-up outcome}

Angiographic follow-up was obtained in two patients with cure. For one patient, patency of residual DAVF after endovascular treatment of a TSS DAVF on the left side and tinnitus was stable, another five patients were cured at clinical follow-up.

\section{Illustrative cases}

\section{Patient 23}

A 56-year-old man with headaches and pulsatile tinnitus demonstrated a left TSS Cognard Type IIa DAVF (Figure 3). An ophthalmological examination revealed bilateral papilledema (Figure 3A). Cerebral angiography demonstrated multiple feeders arising from the left ECA and ICA (Figure 3B) and basal artery system (Figure 3C) with a parallel venous channel. One transarterial and one transvenous embolization of the TSS DAVF were performed. These procedures and occlusion of the ECA feeders significantly reduced the size of the fistula (Figure 3E,F). After transvenous embolization of the left TSS, control angiography demonstrated obliteration of the parallel channel, with preservation of the patent parent sinus (Cognard Type I). During the subsequent months the patient?s tinnitus was in stable condition and no further neurological symptoms were noted, ophthalmological examination was not available.

\section{Discussion}

Depending on their venous drainage patterns, intracranial DAVFs can cause headaches, dementia, chemosis, protosis, bruit, and, rarely, infarction or hemorrhage. The data of our patients confirmed previous studies $7,12,27,38,40,50,56$. The clinical presentation is closely related to the degree of shunting, cerebral venous hypertension, and the pattern of venous drainage, with or without impaired cortical function ${ }^{46}$. Despite spontaneous remission, which occurs in 9.4 to $50 \%$ of cases ${ }^{22}$, treatment is indicated in cases with cortical drainage (Cognard Type IIb or greater), hemorrhage, progressive neurological deficits, or intractable headaches or tinnitus. Although the cure rate of DAVFs by transarterial embolization has been promoted by Onyx, it is limited in smaller arteriovenous fistula DAVFs $^{53}$. Transvenous embolization is still a good option for DAVFs with multiple feeding arteries, especially as they cannot be cured by transarterial embolization ${ }^{19-21}$. Our 23 patients represent $32.76 \%$ (the remaining patients were treated with transarterial embolization or neurosurgery) of the patients who underwent embolization for treatment of DAVFs at our institution in the past ten years. This reflects the evolution of transvenous approaches at a single institution in a period of five years. In our series, the anatomic cure rates were $94.1 \%$ $(16 / 17)$ for DAVFs of the CS and $83.3 \%(1 / 6)$ for DAVFs at other locations. All patients, including those without anatomic cures, experienced improvement.

When catheterization is possible, transvenous embolization is associated with a high rate of permanent long-term occlusion. Occlusion can be achieved with placement of coils via a transvenous route.

\section{Transvenous embolization of $C S$}

There are different transvenous routes to the $\mathrm{CS}$, i.e., by way of the InfPS, contralateral InfPS, basilar plexus or circular sinus, through the $\mathrm{FV}$, angular vein, and SOV, or through the pterygoid plexus ${ }^{2-6,9,14,19,25,27,35,36,42,43,47,52}$. To achieve complete occlusion, the cavernous sinus was tightly packed with GDC. This tight packing may have accounted for the intense nausea and vomiting. Transient VIth or III ${ }^{\text {rd }}$ nerve palsy following coil embolization for cavernous DAVF are well known events ${ }^{2}$. The complex nature of the fistula, the unexpected difficulty in the placement of detachable coils, and our previous experience with Onyx in the treatment of DAVFs promoted us to use Onyx in the treatment of our patien ${ }^{19,20,38}$.

\section{Transvenous embolization of DAVFs in other locations}

For DAVFs in the ACF, we prefer the transvenous procedure with the softest EDC and free coils to fit the draining frontal veins and to minimize the risk of damage and rupture of the frontal veins, appreciating the risk of visual compromise from embolic occlusion of the central retinal artery. Although the venous approach through the elongated, ectactic and potentially fragile pial veins is considered difficult and risky, several cases of tentorial DAVF have been treated by transvenous embolization ${ }^{9,21,23}$.

If the affected TSS is isolated or exhibits prominent retrograde drainage to the cortical 
veins and is not a functional part of the venous circulation, then sinus occlusion can be performed via an endovascular procedure. There was anterograde flow in the vein of Labbe in one case of a lateral sinus fistula, after transvenous embolization of the TSS with sparing of the vein of Labbe.

Kubo et $\mathrm{Al}^{30}$ concluded, on the basis of their three cases and a review of the literature, that second fistulae can occur after complete embolization, with latency periods of more than one year and this finding was confirmed by Kiyosue et $\mathrm{Al}^{26}$. For this reason, we occluded all main feeding arteries prior to obliteration of the affected sinus in our cases with DAVFs of the tentorium and TSS to prevent development of cortical venous reflux and intracranial hem- orrhage. In one patient with a TSS DAVF with a parallel venous channel of the transverse/sigmoid sinus converted a Cognard Type IIa DAVF into a Cognard Type I DAVF with a patent parent sinus.

\section{Conclusions}

Transvenous treatment of intracranial DAVFs can be a highly effective method if various transvenous approaches are attempted. Onyx is a promising embolic agent for the transvenous treatment of DAVFs, and its physical properties warrant further appraisal in larger series of patients. The advantages make this an attractive alternative to various platinum coils already described.

\section{References}

1 Abrahams JM, Begley LJ et Al: Alternative management considerations for ethmoidal dural arteriovenous fistula. Surgical Neurology 58(6): 410-417, 2002.

2 Aihara N, Mase M et Al: Deterioration of ocular motor dysfunction after transvenous embolization of dural arteriovenous fistula involving the cavernous sinus. Acta Neurochir 141: 707-710, 1999.

3 Arat A, Cekirge S et Al: Transvenous injection of Onyx for casting of the cavernous sinus for the treatment of a carotid-cavernous fistula. Neuroradiology 46: 1012 1015, 2004

4 Barrow DL, Sector RH et Al: Classification and treatment of spontaneous carotid cavernous fistula. J Neurosurg 62: 248-256, 1985.

5 Benndorf G, Bender A et Al: Treatment of a cavernous sinus dural arteriovenous fistula by deep orbital puncture of the superior ophthalmic vein. Neuroradiology 43: 499-502, 2001.

6 Benndorf G, Bender A et Al: Transvenous occlusion of dural cavernous sinus fistulas through the thrombosed inferior petrosal sinus: report of four cases and review of the literature. Surg Neurol 54: 42-54, 2000.

7 Benndorf G, Schmidt S et Al: Tentorial dural arteriovenous fistula presenting with various visual symptoms related to anterior and posterior visual pathway dysfunction: case report. Neurosurgery 53: 222-227, 2003.

8 Cognard C, Gobin YP et Al: Cerebral dural arteriovenous fistulas: clinical and angiographic correlation with a revised classification of venous drainage. Radiology 194: 671-680, 1995.

9 Deasy NP, Gholkar AR et Al: Tentorial dural arteriovenous fistulas: endovascular treatment with transvenous coil embolization. Neuroradiology 41: 308-312, 1999.

10 Deshmukh VR, Chang S et Al: Bilateral ethmoidal dural arteriovenous fistulas: a previously unreported entity: case report. Neurosurgery 57(4): E809, 2005.

11 Defreyne L, Vanlangenhove P et Al: Transvenous embolization of a dural arteriovenous fistula of the anterior cranial fossa: preliminary results. Am J Neuroradio 21: 761-765, 2000.

12 Deshmukh VR, Maughan PH, Spetzler RF: Resolution of hemifacial spasm after surgical obliteration of a tentorial arteriovenous fistula: case report. Neurosurgery 58(1): E202, 2006

13 Fujita A, Tamaki N et Al: A tentorial dural arteriovenous fistula successfully treated with interruption of leptomeningeal venous drainage using microvascular Doppler sonography: case report. Surg Neurol 56: 5661, 2001.

14 Goldberg RA, Goldey SH et Al: Management of cavernous sinus-dural fistulas. Indications and techniques for primary embolization via the superior ophthalmic vein. Arch Ophthamol 115(6): 823-824, 1997.

15 Halbach VV, Higashida RT et Al: Dural arteriovenous fistulas supplied by ethmoidal arteries. Neurosurgery 26: 816-823, 1990.

16 Iwamuro Y, Nakahara I et Al: Tentorial dural arteriovenous fistula presenting symptoms due to mass effect on the dilated draining vein: case report. Surg Neurol 65: 511-515, 2006.

17 Jahan R, Gobin YP et Al: Transvenous embolization of a dural arteriovenous fistula of the cavernous sinus through the contralateral pterygoid plexus. Neuroradiology 40: 189-193, 1998.

18 Jesus OD, Rosado JE: Tentorial dural arteriovenous fistula obliteration using the petrosal approach. Surg Neurol 51: 164-167, 1999.

19 Jiang C, Lv X et Al: Transvenous Embolization of Cavernous Sinus Dural Arteriovenous Fistula with Onyx18 and Plentinum coils: Technical Note. NRJ-Neuroradiol J 20(3): 47-52, 2007.

20 Jiang C, Lv X et Al: Transvenous embolization with Onyx for dural arteriovenous fistula of cavernous sinus: a report of two case reports. NRJ-Neuroradiol J 20(6): 718-725, 2007.

21 Jiang C, Lv X et Al: Transarterial and transvenous embolization for tentorial dural arteriovenous fistula: case report. NRJ-Neuroradiol J 20(6): 726-729, 2007.

22 Kai Y, Hamada J et Al: Treatment of cavernous sinus dural arteriovenous fistulas by external manual carotid compression. Neurosurgery 60: 253-258, 2007. 
23 Kajita Y, Miyachi S et Al: A dural arteriovenous fistula of the tentorium successfully treated by intravascular embolization. Surg Neurol 52: 294-298, 1999.

24 Kallmes DF, Jensen ME et Al: Percutaneous transvenous coil embolization of a Djindjian type 4 tentorial dural arteriovenous malformation. Am J Neuroradiol 18: 673-676, 1997

25 Kazekawa K, Iko M et Al: Dural AVFs of the cavernous sinus: transvenous embolization using a direct superficial temporal vein approach. Radiation Medicine 21(3): 138-141, 2003

26 Kim MS, Han DH et Al: Posterior fossa hemorrhage caused by dural arteriovenous fistula: case reports. Surg Neurol 59: 512-517, 2003.

27 Kim DJ, Kim DI et Al: Results of transvenous embolization of cavernous dural arteriovenous fistula: A single-center experience with emphasis on complications and management. Am J Neuroradiol 27(10): 2078-2082, 2006.

28 Kiyousue H, Tanoue S et Al: Recurrence of dural arteriovenous fistula in another location after selective transvenous coil embolization: Report of two cases. Am J Neuroradiol 23: 689-692, 2002.

29 Komotar RJ: Clinicoradiological review: bilateral ethmoidal artery dural arteriovenous fistulas. Neurosurgery 60(1): 131-136, 2007.

30 Kong DS, Kwon $\mathrm{KH}$ et $\mathrm{Al}$ : Combined surgical approach with intraoperative endovascular embolization for inaccessible dural arteriovenous fistulas. Surg Neurol 68: 72-78, 2007.

31 Krisht AF, Burson T: Combined pretemporal and endovascular approach to the cavernous sinus for the treatment of carotid-cavernous dural fistulas: technical case report. Neurosurgery 44(2): 415-418, 1999.

32 Kubo M, Kuwayama N et Al: Dural arteriovenous fistulas developing at different locations after resolution of previous fistulas: Report of three cases and review of the literature. Am J Neuroradiol 23: 787-789, 2002.

33 Kuwayama N, Endo S et Al: Surgical transvenous embolization of a cortically draining carotid cavernous fistula via a vein of the sylvian fissure. Am J Neuroradiol 19(7): 1329-1332, 1998.

34 Lawton M, Chun J et Al: Ethmoidal dural arteriovenous fistule: an assessment of surgical and endovascular management. Neurosurgery 45(4): 805, 1999

35 Lefkowitz M, Giannotta SL et Al: Embolization of neurosurgical lesions involving the ophthalmic artery. Neurosurgery 43(6): 1298-1303, 1998.

36 Leonard F, Jeffrey B, Nicholas JV: Cavernous sinus fistulas: carotid cavernous fistulas and dural arteriovenous malformations. Current Neurology \& Neuroscience Reports 3(5): 415-420, 2003.

37 Liu HM, Huang YC et Al: Transarterial embolization of complex cavernous sinus dural arteriovenous fistulas with low-concentration cyanoacrylate. Neuroradiology 42: 766-770, 2000.

$38 \mathrm{Lv}$ X, Li Y, Wu Z: Endovascular treatment of the anterior cranial fossa dural arteriovenous fistulas. Neuroradiology 50: 433-437, 2008.

39 Masahiro S, Hiroki $\mathrm{K}$ et $\mathrm{Al}$ : Stereotactic radiosurgery for tentorial dural arteriovenous fistulas draining into the vein of Galen: reports of two cases. Neurosurgery 46(3): 730, 2000

40 Matsushige T, Nakaoka M et Al: Tentorial dural arteriovenous malformation manifesting as trigeminal neuralgia treated by stereotactic radiosurgery: a case report. Surg Neurol 66: 519-523, 2006.

41 Mayfrank L, Reul J et Al: Microsurgical interhemispheric approach to dural arteriovenous fistulas of the floor of the anterior cranial fossa. Minimally Invasive Neurosurgery 39(3): 74-77, 1996.
42 Nakamura M, Tamaki N et Al: Selective transvenous embolization of dural carotid-cavernous sinus fistulas with preservation of sylvian venous outflow. Report of three cases. J. Neurosurg 89(5): 825-829, 1998.

43 Oishi $\mathrm{H}$, Arai $\mathrm{H}$ et Al: Complications associated with transvenous embolization of cavernous dural arteriovenous fistula. Acta Neurochir 141: 1265-1271, 1999.

44 Pannu Y, Shownkeen H et Al: Obliteration of a tentorial dural arteriovenous fistula causing spinal cord myelopathy using the cranio-orbital zygomatic approach. Surg Neurol 62: 463-467, 2004.

45 Patrik RT, Harry JC et Al: Evolution of the management of tentorial dural arteriovenous malformations. Neurosurgery 52(4): 750-762, 2003.

46 Paula-Lucas C, Pereica-Caldas J-G-M, Prandini MN: Do leptomeningeal venous drainage and dysplastic venous dilation predict hemorrhage in dural arteriovenous fistula? Surg Neurol 66: S3:2-S3:6, 2006.

47 Quinones D, Duckwiler G et Al: Embolization of dural cavernous fistulas via superior ophthalmic vein approach. Am J Neuroradiol 18(5): 921-928, 1997.

48 Roy D, Raymond J: The role of transvenous embolization in the treatment of intracranial dural arteriovenous fistulas. Neurosurgery 40(6): 1133-1144, 1997.

49 Satomci J, Satoh K et Al: Angiographic changes in venous drainage of cavernous sinus dural arteriovenous fistulas after palliative transarterial embolization or observational management: a proposed stage classification. Neurosurgery 56: 494-502, 2005.

50 Siekmann R, Weber W et Al: Transvenous treatment of a dural arteriovenous fistula of the transverse sinus by embolization with platinum coils and Onyx HD 500+. Interventional Neuroradiology 11(3): 281-286, 2005.

51 So-Hyang I, Chang WO, Dae HH: Surgical management of an unruptured dural arteriovenous fistula of the anterior cranial fossa: natural history for 7 years. Surg Neurol 62: 72-75, 2004

52 Suzuki S, Lee DW et Al: Transvenous treatment of spontaneous dural carotid-cavernous fistulas using a combination of detachable coils and Onyx. Am J Neuroradiol 27(6): 1346-1349, 2006.

53 Toulgoat F, Mounayer C et Al: Transarterial embolization of intracranial dural arteriovenous malformations with ethylene vinyl alcohol copolymer (Onyx-18). J Neuroradiol 33(2): 105-114, 2006

54 Troffkin NA, Graham CB et Al: Combined transvenous and transarterial embolization of a tentorial-incisural dural arteriovenous malformation followed by primary stent placement in the associated stenotic straight sinus. Case report. J. Neurosurg 99(3): 579-583, 2003.

$55 \mathrm{Tu}$ YK, Liu HM, Hu SC: Direct surgery of carotid cavernous fistulas and dural arteriovenous malformations of the cavernous sinus. Neurosurgery 41(4): 798-806, 1997.

56 Urtasun F, Biondi A et Al: Cerebral dural arteriovenous fistulas: percutaneous transvenous embolization. Radiology 199(1): 209-217, 1996.

57 Watanabe T, Matsumaru Y et Al: Multiple dural arteriovenous fistulas involving the cavernous and sphenoparietal sinuses. Neuroradiology 42: 771-774, 2000.

58 White DV, Sincoff EH, Abdulrauf SI: Anterior ethmoidal artery: mirosurgical anatomy and ethmoidal considerations. Neurosurgery 56(4) ONS(2): 406-410, 2005.

Zhongxue $\mathrm{Wu}$

Beijing Neurosurgical Institute

No.6, Tiantan Xili, Chongwen, Beijing

100050 P.R.China

E-mail: ttyyzjb@sina.com 